Ann. Abeille, 1967, 10 (2), I03-II7.

\title{
ÜBER DIE VERERBUNG DER PIGMENTIERUNG BEI ARBEITSBIENEN VON APIS MELLIFICA L. UND IHRER KREUZUNGEN
}

\author{
I. KULINCEVIC (1) \\ Institut für Landwirtschaftliche Zoologie und Bienenkunde \\ der Rheinischen Friedrich-Wilhelm-Universität, Bonn (Allemagne)
}

\section{EINLEITUNG}

Die Vererbung der Farbmuster bei Bienenrassen und ihren Kreuzungen ist eine bis heute oft behandelte Frage. Das gilt besonders fuir Kreuzungen der Carnica $\times$ Ligustica, Mellifica $\times$ Ligustica und Kaukasika $\times$ Ligustica. Noch bestehen Unklarheiten über farbliche Dominanz und matrokline Vererbung. Erst durch die Verwendung der künstlichen Besamung der Königinnen sind die erforderlichen Voraussetzungen für eine exakte Untersuchung dieser Frage gegeben.

Mit dieser Arbeit wird ein Versuch unternommen, das Problem zu klären und einige Missverständnisse zu beseitigen.

Ich werde mich bemühen, eine Übersicht über die bestehende Literatur in dieser Hinsicht zu geben. Die älteren Arbeiten entstanden unter anderen Versuchsbedingungen, da man irrtümlich annahm, die Königin könne sich nur mit einem Drohn paaren. Ausserdem waren die Belegstellen nicht genug isoliert, so dass sich fast immer fehlgepaartes Material anstelle der erforderlichen Kreuzungen ergab.

SLADEN (I9r3) fand nach einer Kreuzung der " goldenen " Königin mit einem "schwarzen " Drohn, dass die Arbeiterinnen farblich intermediär waren. NEwEL, (I9I5) kreuzte italienische Königinnen mit Carnica-Drohnen in einem isolierten Belegstand und erhielt eine Generation $F_{1}$-Arbeiterinnen, die " identisch " mit reinrassigen italienischen Bienen waren. ARMBRUster (I923) untersuchte Kreuzungen zwischen Ligustica und Nigra sowie Ligustica und Fasciata. Die $\mathrm{F}_{1}$-Generation war in einigen Fällen intermediär und in anderen zweigipfelig mit grosser Variabilität. Watson (I927) paarte durch künstliche Besamung eine schwarze Königin mit einem

(') Z. Zt. The Ohio State Universily, Deparlement of Zoology and Entomology. Columbus, Ohio, 432 I0 - U.S. A. 
gelben Drohn und erhielt nur schwarze Bienennachkommenschaft. MichaILofF (I930) fand heraus, dass, indem er Königinnen der zentral-russischen schwarzen Bienenrasse mit goldfarbenen italienischen Drohnen kreuzte " gelb " die dominante Farbe bei go p. Ioo der Nachkommenschaft war. GoETzE (I956) stellte fest, dass nach der Kreuzung zwischen goldenen und dunklen Bienen eine intermediäre $\mathrm{F}_{1}$-Generation vorkam. Not,AN (I937) erhielt ebenfalls eine intermediäre $F_{-1}$ Generation aus der Kreuzung Kaukasika $\times$ Ligustica. FARCAs (I938) sagte, dass die Bastard-Arbeiterinnen, die aus der Kreuzung zwischen einheimischen Königinnen und italienischen Drohnen hervorgingen, in der ersten Generation eine intermediäre Farbe mit einer schwachen Tendenz zur schwarzen Pigmentierung zeigten. RoBERTs (I950) kreuzte - unter Verwendung von künstlicher Besamung -- zwei sehr stark ingezïchtete Linien, eine ganz dunkle und eine fast vollständig gelbe. Fir hat eine Nachkommenschaft gewonnen, die intermediär war. KUNZE (I956) machte eine vergleichende Analyse der Variabilität der Merkmale bei der Kreuzung von Carnica $\times$ Ligustica. Sie fand, dass die Variabilität bei dieser Kreuzung viel grösser war, als bei den Ausgangsrassen. Sie hat ebenfalls die Farbe bei beiden Rassen und ihren Kreuzungen untersucht und behauptete, dass die Rassen in der Farbe einheitlich waren. Bei Kreuzungen zeigte sie hier eine unvergleichbar grössere Variation als bei den Ausgangsrassen. Mackensen (I956) erreichte, nach 7 Generationen Inzucht durch künstliche Besamung, dass sich bei den Nachkommen die Unterschiede in der Farbenmusterung auf praktisch Null verringerten. RUTTNER (I957) ist bei einem Versuch so verfahren, dass er bei Kreuzungen neben einer einheitlich dunklen Rasse (var. mellifica oder var. carnica) jeweils eine Rasse mit deutlicher Gelbfärbung des Abdomens und gelben Scutelum bei den Arbeiterinnen verwendete (var. cypria). Die Nachkommen dieser verschiedenen Drohnentypen konnten ohne Schwierigkeiten voneinander unterschieden werden. EI, BANBY (I965) behauptete, dass die Arbeitsbienen der $\mathrm{F}_{1}$-Generation nach der Kreuzung zwischen ägyptischen gelben Königinnen und Carnica-Drohnen identisch mit gelben ägyptischen Bienen gewesen seien. Er sagte : "Aus diesem kann man schliessen, dass die ägyptische Färbung über die carniolische vorherrscht."

Es ist interessant, noch einige Angaben über die Vererbung anderer Merkmale bei der $F_{1}$-Generation der Bienen aufzuzeigen, weil im Zusammenhang mit den Färbungsmustern die Vererbung der Grösse der Gesamtfläche der 3., 4. und 5. Tergit nach betrachtet wird.

KRCZAL (I952) untersuchte die Variabilität von Flügelindex und Rüssellänge bei der Honigbiene und ihren Kreuzungen. Die Werte bei den Kreuzungen zeigten sich als intermediär. RUTTNER (I953) fand bei der $\mathrm{F}_{1}$-Generation der Arbeiterinnen, dass die Mittelwerte des Cubital-Index deutlich von dem zu erwartenden intermediären Typ abweichen. Nach seinen Ergebnissen zeigten die reziproken Kreuzungen jedoch die Werte, die beträchtlich in Richtung des mütterlichen Phänotyps verschoben sind. Es liegt hier also eine partielle matrokline Vererbung vor. DRESCHER und KULINČEvić (I967) - Manuskript unveröffentlicht - haben zwei Rassen (Carnica und Mellifica) unter Verwendung von künstlicher Besamung durch einige Generationen ingezüchtet, gekreuzt und die Rüssellänge untersucht. Die Ergebnisse zeigten, dass die Vererbung des Ruissellänge intermediär und die Variabilität bei den Bastarden nicht grösser als beim Ausgangsmaterial ist. 


\section{MATERIAL UND ME'THODE}

Bei diesem Versuch wurden zwei clurch Färbungsmuster sehr deutlich sich unterscheidende geographische Rassen verwendet :

Apis mellifica var. camica (Ca) und Apis mellifica var. ligustica (L.). Die Linien beider Rassen wurden unter Verwendung künstlicher Besamung ingezüchtet. Bei der Besamung wurde Sperma von einem I)rohn benutzt, auch bei den reziproken Kreuzungen. Als lilternköniginnen fanden für Carnica \& B-77 und für Ligustica o B-69 Verwendung. Die reziproken Kreuzungen bestanden aus of'Tochter von B-77 $\times 3-\mathrm{I})$ rohn von $\mathrm{B}-69 \rightarrow \mathrm{B}$ 102, f-Tochter von B-69 $\times$ j-1)rohn von B-77 $\rightarrow$ B rog.

Zwei Tage nach der Besamung wurden die Königinnen mit $\mathrm{CO}_{2}$ behandelt und in Ableger von drei gut besetzten Normalmass-Waben gesetzt. J)urchschnittlich begann nach r 2 'lagen die Eiablage.

Die verdeckelte Brut wurde 4-5 Tage vor dem Schlüpfen aus den Ablegern entnommen und bei $34-35^{\circ} \mathrm{C}$ zum Schlüpfen in Brutschrank gelassen. Die P'roben (Konservierung in 70 p. 100 Alkohol) wurden mit 5-6 Tage alten Bienen vorgenommen, damit sich die Ausfärbung vollständig zeigen konnte. Mit IHilfe eines scharfen Wasserstrahls konnte das ganze $\Lambda$ bdomen von den inneren Organen und der Muskulatur befreit werden. Durch eine Iagerung in Milchsäure wurden die Tergite weicher, was für die Vorbereitung der Präparate sehr wichtig war. Die Farbmuster wurden an 3., 4. und 5. Tergit untersucht. I)ie Tergite wurden zwischen zwei Objektträgern mit Milchsäure präpariert, so dass die granze T'ergitfläche ausgestreckt war und die einzelnen Tergite danach vorsichtig auseinandergenommen. I ann fand mit IIilfe eines Zeichnungsgerätes eine Fergrösserung ( $889 \times)$ und eine Zeichnung statt. Für die Messungen wurde das l'lanimeter "Aristo " verwendet. Aus jeder Probe ist von je 30 Bienen das Teil-Abdomen (.3., 4. und 5. Tergit) gezeichnet und gemessen worden. Fs wurden cimmal die Gesamtttergit-Flächen, desweiteren die dunkeln und gelben T'eile gemessen. Bei Bastarden sind auch die Flächen mit verïnderten Frundstrukturen und Farbton beachtet und gemessen worden.

Bei der Beurteilung der Fïrbungsmerkmale der Abdominal-Tergite von Arbeitsbienen haben wir streng zu unterscheiden zwischen Fürbungsmuster oder "Farbzeichen " und der Färbungsintensität oder dem " I'arbton ". In der züchterischen l'raxis wird der Farbton zumeist vernachlässigt und nur auf dic Farbzeichen geachtet. In dieser Arbeit sind die Farbzeichen oder die Farbmuster von besonderen Interesse.

Um die Farbmuster bei Arbeitsbienen zu beurteilen, hat man bis jetz fast ausschliesslich die Klassenverteilung verwendet. ARMBRrsTrr (I923) hat ein 9-Klassen-System eingeführt. Er verwendet das 3. 'Tergit als Masstab, Zanibre (1923) ro Klassen, Gomtze (I9.30) für die Tergite 3,4 und 5,9 Klassen, Veccur (1927) sogar 25 Klassen, Klvze (I956) für Bastardbienen aus der Kreuzung I. igustica X Carnica Io Klassen und RoBEkTS (I951) is Klassen. Wie zu sehen ist, haben wir ziemlich viele Klassifizierungen. Der Mangel der angewendeten Klassen-Systeme, besonders für präzise genetische Studien, zeigt sich deutlich. Er liegt in der willkürlichen lïinteilung in Klassen und im Fehlen von präzisen Messungen und Berechnungen. Mit meinem Versuch und der Analyse der lirgebnisse möchte ich keine neue Klassen-skala einführen. Für einen Vergleich werde ich froetzF. (1930) 9-Klassen-Hystem übernehmen.

Diese Intersuchung wurde als lerweiterung ähnlicher Versuche bei I)rohnen von Kirlincević (1966) durchgeführ1 und eine yuantitative Bewertung angest rebt.

\section{ERGEBNISSE UND DISKUSSION}

Die Messwerte in absoluten /ahlen iiber die Farbmuster von beiden Rassen (Linien) sind in Tabelle I gegeben. Ca-Tergite (3., 4. und 5.) sind als vollständig dunkel angerechnet, obwohl am Rande der Seitenlappen und am Vorderrand der 'Tergite aufgehellte, kleine Flächen auftraten. Aber diese etwas helleren Flächen konnte man mit einbeziehen, weil ihre Grundfarbe dunkel oder bräunlich war. Die 'Tergite von allen 30 untersuchten Bienen wurden in GoETzE's Klasse I eingeordnet. Die Variabilität nach Varianz-Analyse zeigte sich unbedeutend, aber beim Vergleich 


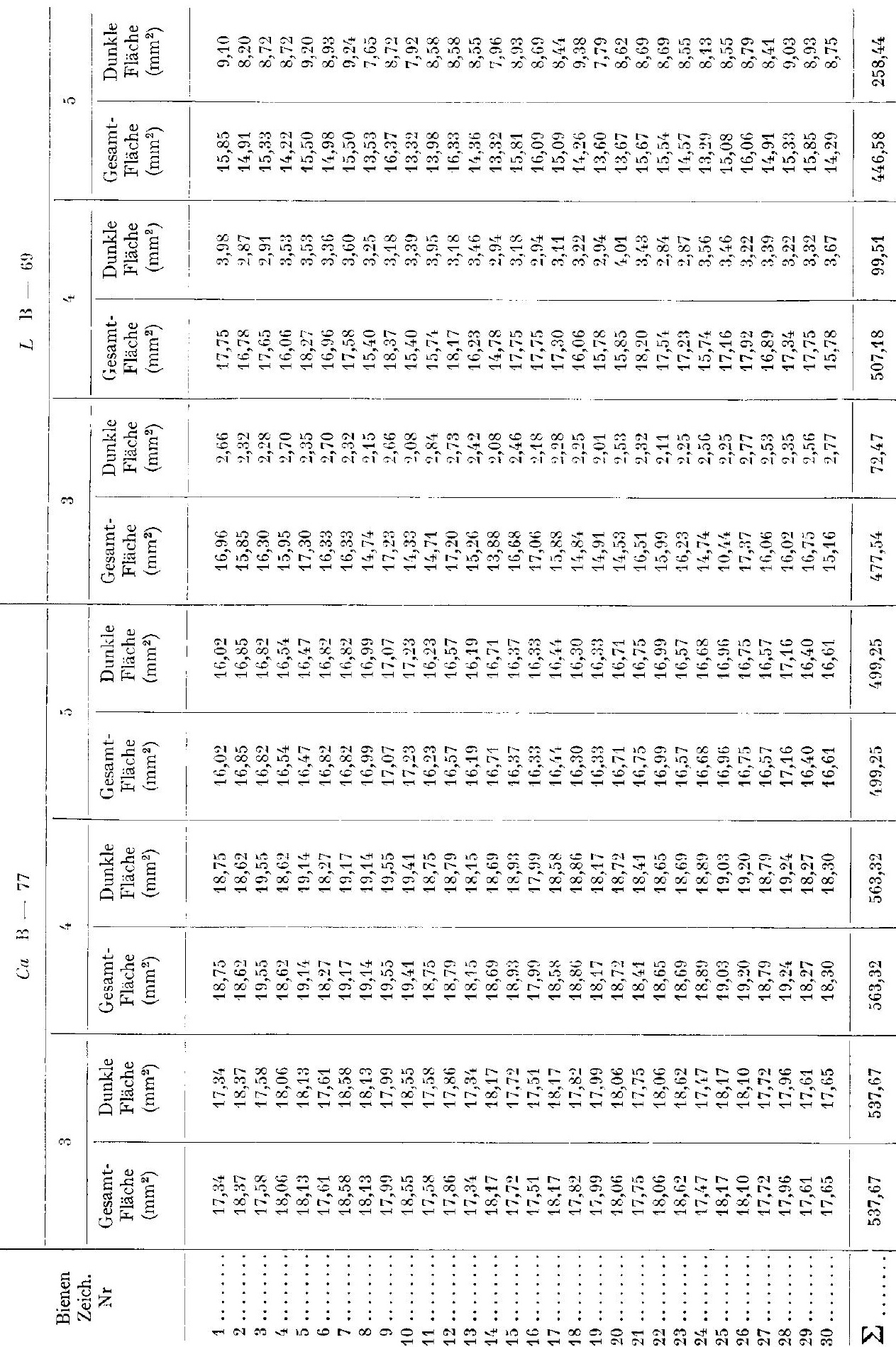


der I)urchschnittswerte im Verhältnis mit $L$ und Bastarden gross genug. Die Durchschnittsgrösse der dunklen Flächen für alle drei Tergite ist $53,34 \mathrm{~mm}^{2}$ und die Streuung $\pm \mathrm{I}, 47 \mathrm{~mm}^{2}$.

Die dunklen Streifen, die die Farbmuster bei $L$ bildeten, waren deutlich von gelben Flächen abgegrenzt und an allen drei Tergiten im Durschschnitt $\mathrm{I} 4,35 \mathrm{~mm}^{2}$ gross Die Streuung war noch geringer als bei $\mathrm{Ca}$; sie betrug $\pm \mathrm{I}, 30 \mathrm{~mm}^{2}$. Die hellen Tergitflächen waren stark mit gelbem Pigment gefärbt und nur die Seitenlappen hatten teilweise farblosen Untergrund. Alle 'Tergitmuster von $L$ gehörten GoETzE's Klasse IX an.
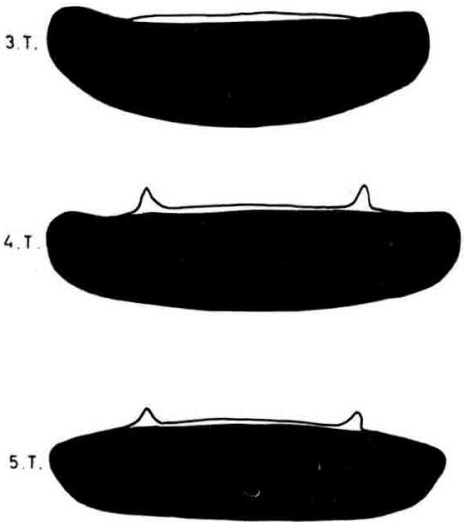

$5 \mathrm{~T}$
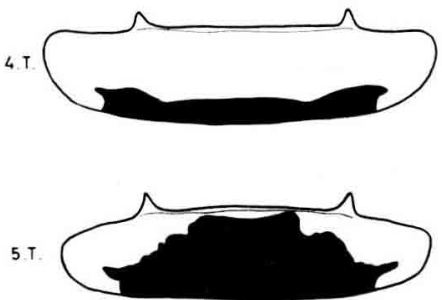
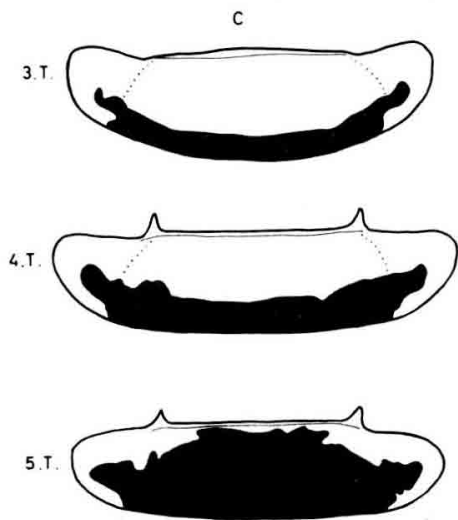

Авв. I. - Zeiclnungsmuster fïr drei Tergite von

A : Carnica

B : Ligustica

C : Carnica $\times$ Ligustica und Ligustica $\times$ Carnica

Fig. 1. - Dessins de 3 tergites des races

A : Carnica

B : Ligustica

C : Carnica $\times$ Ligustica et Ligustica $\times$ Carnica 
Die Messwerte der Gesamt-dunklen und unbestim

Mesures de la superficie totale ainsi que des parties sombres et indéterm

\begin{tabular}{|c|c|c|c|c|c|c|c|c|}
\hline \multirow{3}{*}{$\begin{array}{l}\text { Bienen } \\
\text { Zeich. } \\
\text { Nr }\end{array}$} & \multicolumn{8}{|c|}{$\mathrm{Ca} \times L \quad \mathrm{~B}-102$} \\
\hline & \multicolumn{3}{|c|}{3} & \multicolumn{3}{|c|}{4} & \multicolumn{2}{|c|}{5} \\
\hline & $\begin{array}{l}\text { Gesamt- } \\
\text { Fläche } \\
\left(\mathrm{mm}^{2}\right)\end{array}$ & $\begin{array}{l}\text { Dunkle } \\
\text { Fläche } \\
\left(\mathrm{mm}^{2}\right)\end{array}$ & $\begin{array}{l}\text { Unbest. } \\
\text { Fläche } \\
\left(m^{2}\right)\end{array}$ & $\begin{array}{l}\text { Gesamt- } \\
\text { Fläche } \\
\left(\mathrm{mm}^{2}\right)\end{array}$ & $\begin{array}{l}\text { Dunkle } \\
\text { Fläche } \\
\left(\mathrm{mm}^{2}\right)\end{array}$ & $\begin{array}{l}\text { Unbest. } \\
\text { Fläche } \\
\left(\mathrm{mm}^{2}\right)\end{array}$ & $\begin{array}{l}\text { Gesamt- } \\
\text { Fläche } \\
\left(\mathrm{mm}^{2}\right)\end{array}$ & $\begin{array}{l}\text { Dunkl } \\
\text { Fläch } \\
\text { (mm² }\end{array}$ \\
\hline $1 \ldots \ldots$ & 17,06 & 3,70 & 4,01 & 16,78 & $5,66^{\prime}$ & $4_{4}, 19$ & 15,02 & 10,10 \\
\hline $2 \ldots \ldots$ & 16,68 & 3,74 & 4,22 & 17,61 & 5,88 & 4,08 & 15,81 & 10,69 \\
\hline $3 \ldots \ldots$ & 16,96 & 3,61 & 4,19 & 17,17 & 5,61 & 4,36 & 15,74 & 10,28 \\
\hline $4 \ldots \ldots$ & 16,82 & 3,53 & 3,77 & 17,37 & 5,47 & 4,36 & 15,33 & 10,31 \\
\hline $5 \ldots \ldots$ & 16,61 & 3,67 & 4,12 & 17,82 & 5,92 & 4,80 & 15,19 & 10,38 \\
\hline $6 \ldots \ldots$ & 15,99 & 3,53 & $3,6: 3$ & 17,20 & 5,81 & 4,50 & 15,26 & 10,35 \\
\hline $7 \ldots \ldots$ & 16,71 & 3,98 & 3,88 & 18,03 & 5,61 & 4,60 & 16,54 & 11,14 \\
\hline $8 \ldots \ldots \ldots$ & 16,19 & $: 3,70$ & 3,77 & 17,58 & 6,37 & 5,08 & 15,12 & 10,45 \\
\hline $9 \ldots \ldots$ & 16,09 & 3,63 & 3,77 & 17,51 & 5,16 & 4,12 & 15,36 & 10,14 \\
\hline $10 \ldots \ldots$ & 16,37 & 3,63 & 3,67 & 16,78 & 5,22 & 4,01 & 15,92 & 11,42 \\
\hline $11 \ldots \ldots \ldots$ & 17,40 & 3,95 & 3,81 & 18,62 & 6,06 & 3,49 & 16,61 & 11,07 \\
\hline $12 \ldots \ldots \ldots$ & 16,33 & 3,53 & 3,39 & 17,79 & 5,71 & 4,71 & 16,06 & 10,28 \\
\hline $13 \ldots \ldots$ & 17,16 & 4,29 & 3,53 & 18,17 & 6,82 & 3,74 & 16,19 & 11,66 \\
\hline $14 \ldots \ldots$ & 15,71 & 4,22 & 3,08 & 17,58 & 6,19 & 4,22 & 15,64 & 10,87 \\
\hline $15 \ldots \ldots$ & 17,47 & 4,46 & 3,32 & 19,10 & 7,79 & 4,12 & 17,13 & 12,94 \\
\hline $16 \ldots \ldots \ldots$ & 16,99 & 3,81 & 4,05 & 18,20 & 6,06 & 4,36 & 16,16 & 10,76 \\
\hline $17 \ldots \ldots$ & 16,96 & 3,95 & 3,77 & 18,96 & 5,67 & 5,29 & 16,02 & 10,76 \\
\hline $18 \ldots \ldots$ & 16,99 & 2,81 & 4,19 & 18,03 & 5,61 & 4,33 & 16,16 & 10,87 \\
\hline $19 \ldots \ldots \ldots$ & 15,71 & 3,91 & 3,56 & 17,09 & 6,19 & 3,70 & 15,19 & 10,59 \\
\hline $20 \ldots \ldots$ & 17,02 & 3,95 & 3,53 & $18,2 / t$ & 6,33 & 1,12 & 16,47 & 10,73 \\
\hline $21 \ldots \ldots \ldots$ & 16,82 & 3,32 & 4,15 & 17,58 & 5,54 & 4,29 & 15,95 & 9,90 \\
\hline $22 \ldots \ldots \ldots$ & 16,10 & 3,91 & 3,18 & 17,85 & 6,47 & 3,91 & 15,85 & 10,52 \\
\hline $23 \ldots \ldots \ldots$ & 16,51 & 4,12 & 3,70 & 17,51 & 5,71 & $\{, 17$ & 15,71 & 10,59 \\
\hline $24 \ldots \ldots \ldots$ & 16,92 & 4,39 & $3,3 \geq$ & 17,96 & 6,37 & 4,20 & 15,47 & 11,11 \\
\hline $25 \ldots \ldots \ldots$ & 16,26 & 4,05 & 3,$6 ; 3$ & 17,47 & 5,40 & 4,71 & 15,78 & 10,45 \\
\hline $26 \ldots \ldots \ldots$ & 15,57 & 3,61 & 3,46 & 16,96 & 5,43 & 4,17 & 15,02 & 9,97 \\
\hline $27 \ldots \ldots \ldots$ & 17,16 & $3,7 t$ & 3,91 & 17,85 & 5,57 & 4,20 & 16,09 & 10,52 \\
\hline $28 \ldots \ldots$ & $15,7 x_{1}$ & 3,13 & 3,70 & 16,92 & 5,71 & 4,36 & 14,88 & 9,93 \\
\hline $29 \ldots \ldots$ & 16,78 & 4,01 & 8,98 & 17,72 & $6,6^{\prime} \mathrm{t}$ & 4,05 & 15,02 & 10,59 \\
\hline $30 \ldots \ldots$ & 16,13 & 3,67 & 3,74 & 17,34 & 5,16 & 4,67 & $15, \pm 3$ & 10,48 \\
\hline$\Sigma$ & 497,20 & $11,65^{2}$ & $112,0^{\prime}$ & 530,08 & 177,12 & 127,98 & ${ }_{4}^{4} 72,12$ & 319,85 \\
\hline
\end{tabular}


2.

hen der Arbeiterinnen-Tergifarbmuster bei nreziproken-Kreuzunge $\mathrm{Ca} \times \mathrm{L}$

2

ergites chez les ouvières issues de croisements réciproques $\mathrm{Ca} \times \mathrm{L}$

I. $\times$ C $\mathrm{Ca} \quad \mathrm{B} .-\mathrm{L} 0 \mathrm{9}$

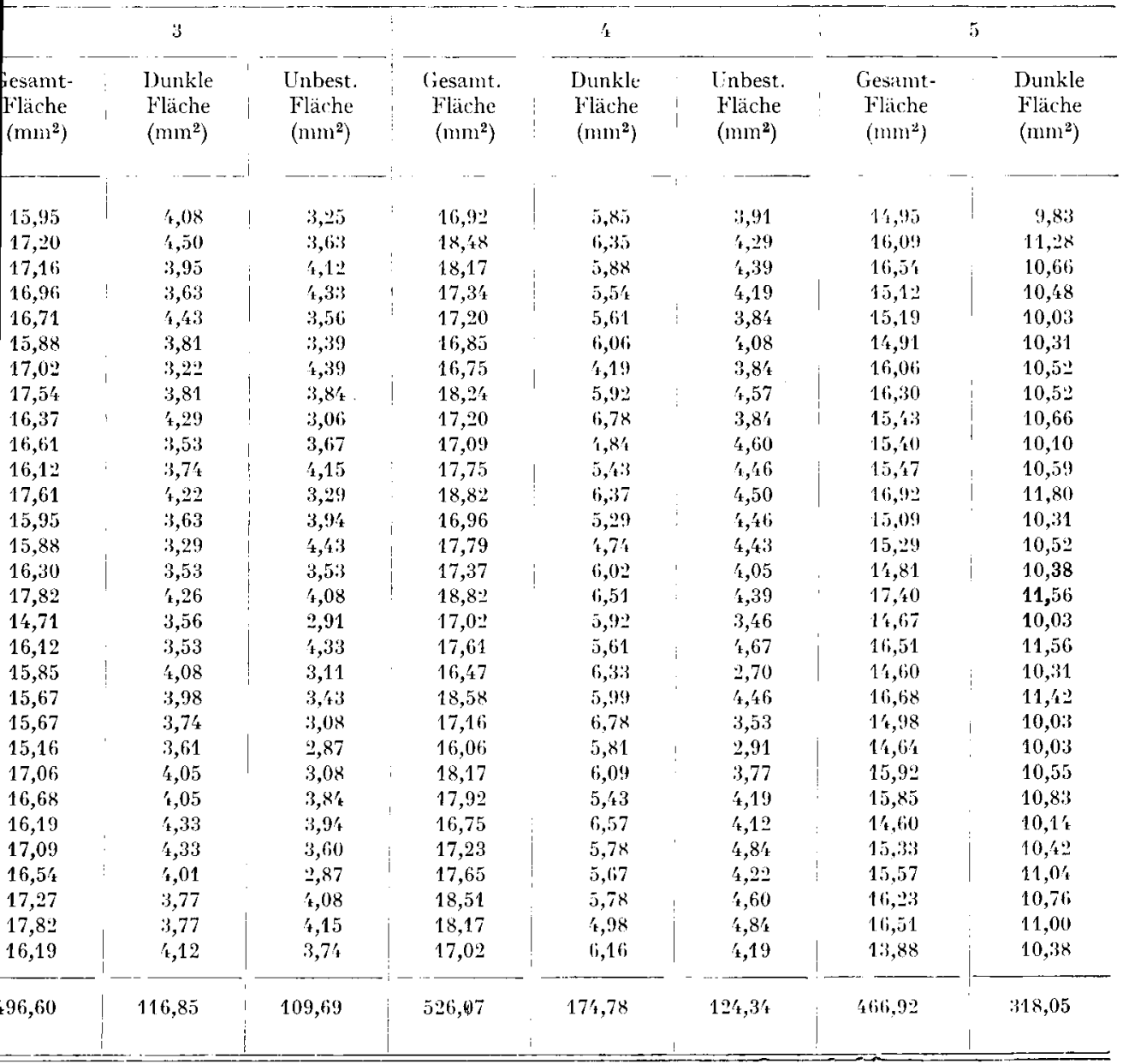


Die Zeichnungen $\mathrm{Ab}$. IA und IB zeigen die Farbmuster von $C a$ und $L$, die als repräsentative Beispiele gelten können. Die Messwerte für die reziproken Kreuzungen $C a \times L$ sind in Tabelle 2 aufgezeigt. Die Gesamtdunkelfläche bei Reziproken hat sich im Verhältnis zu $L$ deutlich vergrössert. Das ist aus den Durchschnittswerten leicht erkennbar, $C a \times L 20,38 \mathrm{~mm}^{2}$ und $L \times C a 20,38 \mathrm{~mm}^{2}$. Diese Durchschnitte unterscheiden sich nach $t$-Test-Methode nicht voneinander (Tab. 3). Die Zeichnung Ab. IC zeigt die Farbmuster von reziproken Kreuzungen. Die Streunng bei Bastarden ist im Vergleich mit $L$ grösser geworden. Zwischen $C a$ und $L \times C a$ ist kein Unterschied in der Streuung festzustellen. Bei Bastarden zeigt sich am Seitenlappen die veränderte Grundstruktur und der Farbton. 'Teilweise sind noch Spuren von gelbem Pigment zu sehen, jedoch beeinflusst diese Veränderung nicht die Farbmuster, weil nur klar gefärbte Streifen für die Beurteilung des Farbmusters in Betracht gezogen wurden. Eine Finstufung in GoETzE's Klassen-System für alle Muster von reziproken Bastarden ist anschliessend in Klasse VIII gegeben d.h., in der benachbarten Klasse

TABELLE: 3

Vergleich der Varianzen und Durchschnitle für die dunkel pigmentierten Flächen dreier Tergite

TABI,EAU 3

Comparaison des variances et moyennes de la surface pigmentée sombre du $3 \mathrm{e}$ tergite

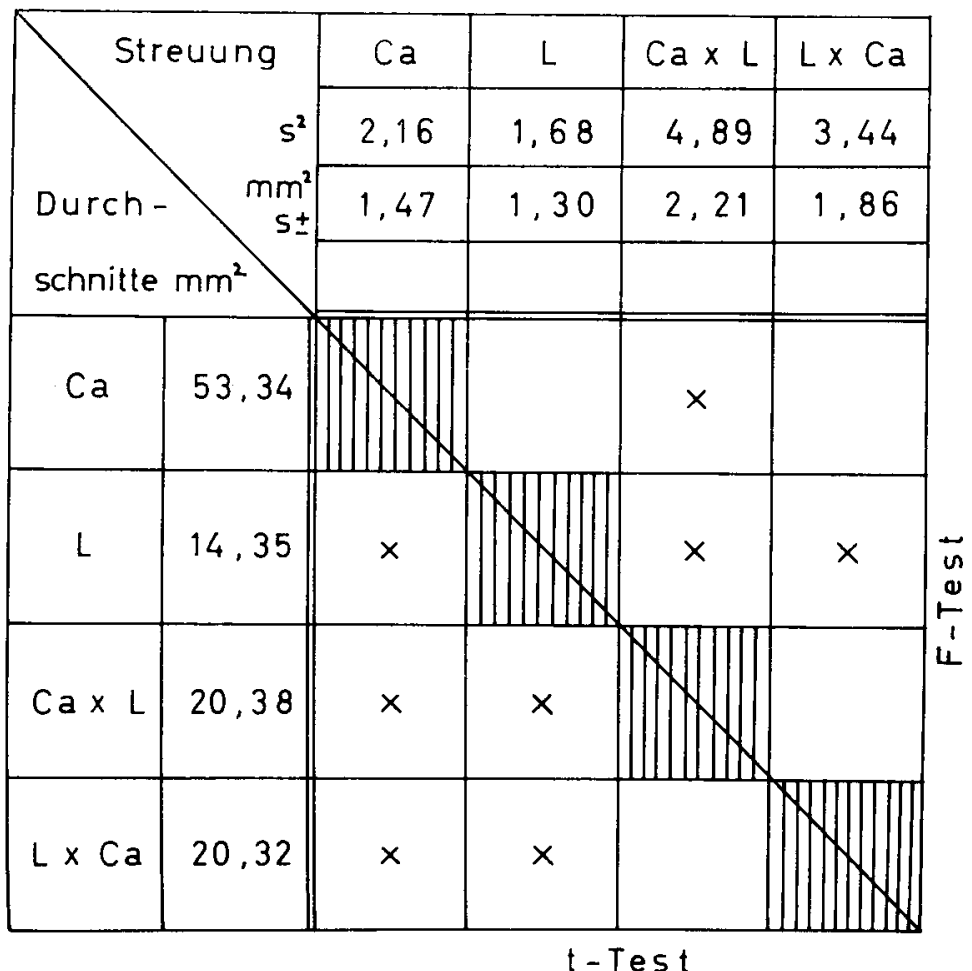


der $L$-Linie. Diese Einstufung und die Messergebnisse mit statistischen Berechnungen zeigen deutlich, dass es sich hier nicht um eine intermediäre Vererbung von Farbmustern handelt-I)as Dominieren der gelben Zeichenmuster ist klar erkennbar.

Einen Vergleich der Varianzen und I)urchschnitte der Farbmuster nur für das 3. 'Tergit von $C a$ und $L$ und ihren reziproken Kreuzungen finden wir in Tabelle 4. Die Varianzen und Durchschnitte zeigen die gleiche Tendenz wie beim Vergleich die Gesamtdunkelflächen d.h., auch hier unterscheiden sich die Reziproken nicht voneinander. Wenn wir die absoluten Werte der dunklen Fläche des 3. Tergits der Reziproken mit den $L$ Mustern vergleichen, sehen wir, dass keine der Reziproken-Tergite den gleichen Wert hat oder im Bereich von $L$ Mustern zu finden ist. Die Werte überschneiden sich nicht.

TABEI,I,E 4

Vergleich der Varianzen und Durchschnitte für die dunkel pigmentierte Fläche des 3. Tergits

TABLEAU 4

Comparaison des variances et moyennes de la surface pigmentée sombre du $3^{\mathrm{e}}$ tergite

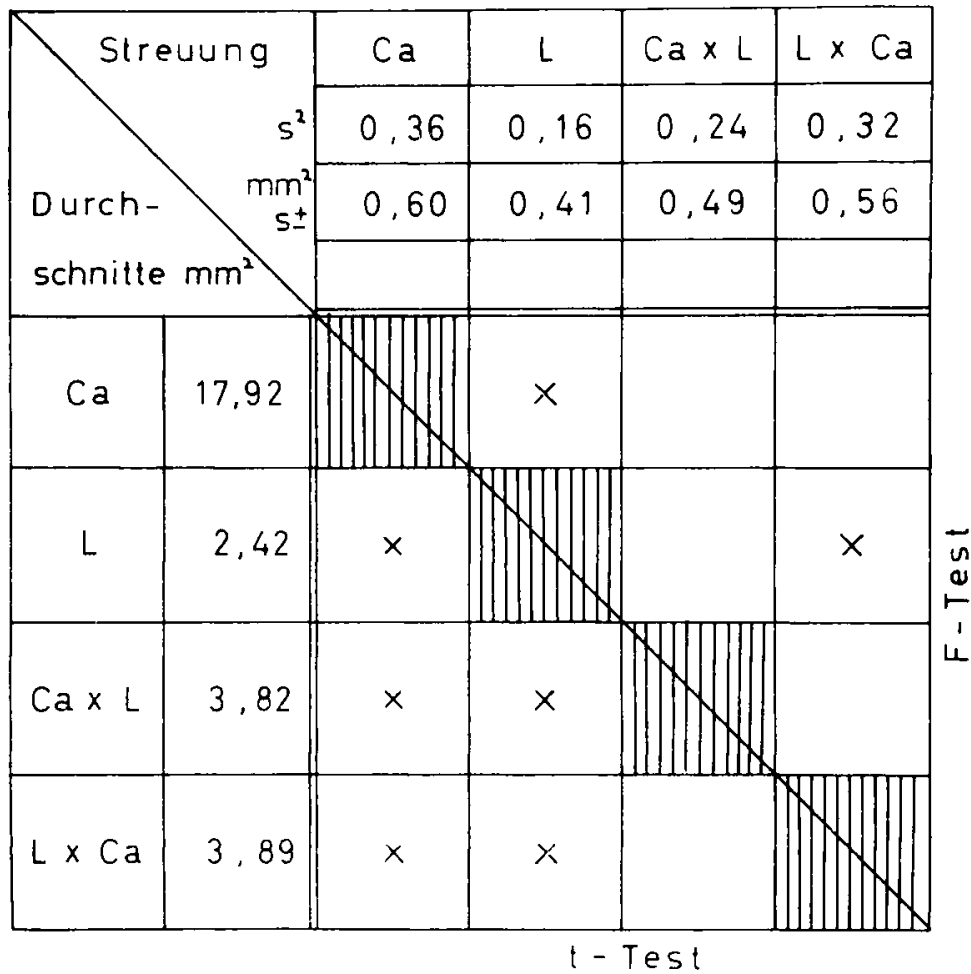

Die Gesamtfläche aller drei Tergite (3., 4. und 5.) wurde auch gemessen und verglichen, (Tabelle 5). Von besonderem Interesse ist, dass die Gesamtfläche der $L$ Tergite eine grössere Streuung zeigt, als die der Gesamtdunkelfläche. 
TABELLE 5

Vergleich der Varianzen und Durchschnitte für die Gesamtfläche dreier Tergile

TABI,EAU 5

Comparaison des variances et moyennes de la surface totale du $3 \mathrm{e}$ tergite

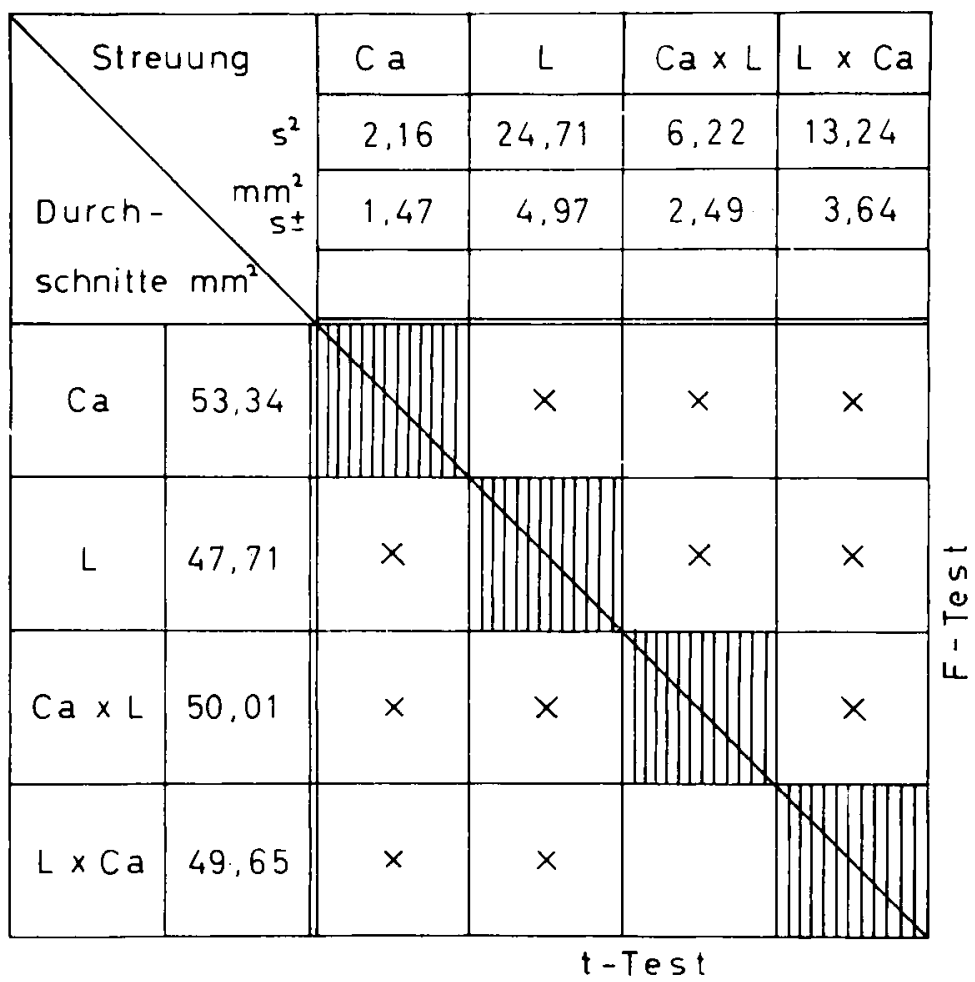

TA

Der prosentuale Anteil dunkler und gelber Flächen der Arbeiterinn

$\mathbf{T}$

Pourcentage des surfaces sombres et jaunes des tergites chez

\begin{tabular}{|c|c|c|c|c|c|c|c|c|c|}
\hline & & $\therefore a$ B & 77 & & & & $B-$ & & \\
\hline Tergite & $\begin{array}{c}\text { Anz. } \\
\text { Bienen }\end{array}$ & $\begin{array}{l}\text { Gesamt- } \\
\text { Fläche } \\
\left(\mathrm{mm}^{2}\right)\end{array}$ & $\begin{array}{l}\text { Dunkle } \\
\text { Tläche } \\
\left(\mathrm{mm}^{2}\right)\end{array}$ & $\begin{array}{c}\text { Dunkle } \\
\text { Flärhe } \\
(\%)\end{array}$ & $\begin{array}{l}\text { Gesaunt- } \\
\text { Fläche } \\
\left(m m^{2}\right)\end{array}$ & $\begin{array}{l}\text { Dunkle } \\
\text { Fläche } \\
(\text { (mm²) }\end{array}$ & $\begin{array}{l}\text { Gelbe } \\
\text { Fläche } \\
\left(\mathrm{mm}^{2}\right)\end{array}$ & $\begin{array}{c}\text { Dumkle } \\
\text { Fläche } \\
\left(\begin{array}{c}0 \\
0\end{array}\right)\end{array}$ & $\begin{array}{c}\text { Gelbe } \\
\text { Fläche } \\
(0) \\
0\end{array}$ \\
\hline & $\ldots$ & $-\ldots$ & - &.- & & & - & & - \\
\hline $3 \ldots$ & 30 & 17,92 & $17,9:$ & 100 & $15,9=$ & 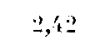 & 13,49 & 15,2 & $8^{\prime \prime}, i^{\prime}$ \\
\hline t... & 30 & 18,78 & 18,78 & 100 & $16, y=$ & $3,3=$ & 13,51 & 19,2 & 3916 \\
\hline $5 \ldots$ & 30 & $16,6^{\prime}$ & $16,6^{\prime} \mathrm{k}$ & 100 & 11,87 & $x, 61$ & 6,26 & 57,9 & 42,1 \\
\hline & & 53,34 & $53,3,3^{\prime}$ & 100 & $\{7,71$ & 14,35 & 33,36 & 30,1 & 69,9 \\
\hline
\end{tabular}


Die reziproken Kreuzungen stehen in der Grösse intermediär und die Durchschnitte unterscheiden sich nicht voneinander, jedoch ist die Streuung bei $L \times C a$ grösser als bei $\mathrm{Ca} \times L$. Die prozentuale Zunahme der dunklen Pigmentierung bzw. Musterung bei einzelnen Tergiten und der Gesamtdunkelfäche ist aus Tabelle $6 \mathrm{zu}$ entnehmen. Der Anteil von dunkler Pigmentierung bei Reziproken vergrössert sich durchschnittlich um 10,7 p. I00, bei dem 3. Tergit un 8,0 p. roo, 4. Tergit um 13,8 p. Ioo und bei dem 5. Tergit um Io,7 p. Ioo.

Ich möchte mit einigen Worten die vorliegenden Ergebnisse über die Vererbung der Pigmentierung bzw. der Farbmuster mit den Ergebnissen der anderen Autoren vergleichen, bestehende Unterschiede aufzeigen und evtl. klären.

Die Farbmuster bei der $\mathrm{F}_{\mathrm{j}}$-Generation aus reziproken Kreuzungen $\mathrm{Ca} \times L$ sind nach meinen Messungen und Berechnungen nicht identisch mit $L$ wie bei NEWELL (I9I5) oder Ei, Banby (I965) (Kreuzung Carnica $\times$ Fasciata). sie sind nicht intermediär wie bei Staden (I9I3), Nolan (I937) und Goetze (1936), ebenfalls nicht intermediär mit schwachem, schwarzen Dominieren wie bei FARCAS (I938), nicht uneinheitlich wie bei ARMBRUSTER (I923) und nicht sehr stark variabel von "dreiringig gelb bis vollstöndig schwarz" wie bei KUNZE (I956), auch sind sie nicht schwarz wie bei Warson (I927). Das Dominieren der gelben Farbe von 90 p. Ioo der $\mathrm{F}_{1}$-Generation bei MrCHAILOFF (I930) ist nicht ohne weiteres anzunehmen. ROBERTS (I950) hatte kein Material, das eine stabile geographische Rasse vertritt ; er hatte nur eine sehr extreme Inzucht, besonders bei der $L$ Linie. Deswegen ist es nicht leicht, mit seinen Ergebnissen einen Verglleich zu ziehen. Ausserdem hat RoBERTs nur eine Klassifikation nach Ähnlichkeit gemacht und es ist nicht klar, ob er die Zeichnungsmuster als Merkmal betrachtete oder auch noch die von mir erwähnten Eintrübungen am Seitenlappen der Tergite. Eit beurteilte die Pigmentierung der ganzen Abdomina. Bei meiner Analyse wurden die 3., 4. und 5. Tergit beriicksichtigt.

Wie aus den Ergebnissen zu ersehen ist, hat sich bei dem Material, das ich untersuchte, die gelbe Musterung als dominant gezeigt, aber eine Steigerung von dunklen Streifen ist statistisch gesichert. Die $F_{1}$-Generation ist einheitlich mit etwas LE 6

rgitfarbmuster bei $\mathrm{Ca}$ und $\mathrm{L}$, sowie bei resiproken Kreuzungen $\mathrm{Ca} \times \mathrm{L}$

U 6

zrières $\mathrm{Ca}$ et $\mathrm{L}$ et issues des croisements réciproques $\mathrm{Ca} \times \mathrm{L}$

\begin{tabular}{|c|c|c|c|c|c|c|c|c|c|}
\hline \multicolumn{4}{|c|}{$\mathrm{Ca} \times \mathrm{L} \quad \mathrm{B}-102$} & & \multicolumn{4}{|c|}{$L \times C a$} & \multirow[b]{2}{*}{$\begin{array}{c}\text { Gelbe } \\
\text { Fläche } \\
(\%)\end{array}$} \\
\hline $\begin{array}{l}\text { Gesamt- } \\
\text { Fläche } \\
\left(\mathrm{mm}^{2}\right)\end{array}$ & $\begin{array}{l}\text { Dunkle } \\
\text { Fläche } \\
\left(\mathrm{mm}^{2}\right)\end{array}$ & $\begin{array}{l}\text { Gelbe } \\
\text { Fläche } \\
\left(\mathrm{mm}^{2}\right)\end{array}$ & $\begin{array}{c}\text { Dunkle } \\
\text { Fläche } \\
(\%)\end{array}$ & $\begin{array}{c}\text { Gelbe } \\
\text { Fläche } \\
(\%)\end{array}$ & $\begin{array}{l}\text { Gesamt- } \\
\text { Fläche } \\
\left(\mathrm{mm}^{2}\right)\end{array}$ & $\begin{array}{l}\text { Dunkle } \\
\text { Fläche } \\
\left(\mathrm{mm}^{2}\right)\end{array}$ & $\begin{array}{l}\text { Gelbe } \\
\text { Fläche } \\
\left(\mathrm{mmi}^{2}\right)^{2}\end{array}$ & $\begin{array}{c}\text { Dunkle } \\
\text { Fläche } \\
(\%)\end{array}$ & \\
\hline 16,56 & 3,82 & 12.77 & 23,0 & 77,0 & $16,5 / \mathrm{k}$ & 3,89 & 12,66 & 23,1 & 76,6 \\
\hline 17,71 & 5,91 & 11,80 & 33,4 & 66,6 & 17,54 & 5,85 & 11,69 & 33,3 & 66,7 \\
\hline 15,74 & 10,65 & 5,09 & 67,7 & 32,3 & 15,57 & 10,58 & 4,98 & 68,0 & 32,0 \\
\hline 50,01 & 20,38 & 29,66 & 40,7 & 59,3 & 49,655 & 20,32 & 29,34 & 40,9 & 59,1 \\
\hline
\end{tabular}

Annales de l'Abeille. - 1967. 
grösserer Streuung als beim Ausgangsmaterial in Hinsicht auf die dunkle Fläche bei $L$. Es zeigt sich keine Matroklinie, weil zwischen reziproken Hybriden kein gesicherter Unterschied der Varianzen bestand und die Durchschnittsvergleiche keinen Hinweis dafür zeigten.

Woher stammen die grossen Unterschiede in den Befunden? Ich vermute folgendes : Wie betont wurde, sieht bei oberflächlicher Betrachtung die $F_{1}$-Generation $C a \times L$ ähnlich aus wie $L$ und ist einheitlich wie sie. Erst die genauen Untersuchungen und Messungen zeigen einen Unterschied. I)aher stammen wahrscheinlich die Behauptungen von NewEI, (I9I5) und Ii, BANBy (I965), dass die $\mathrm{F}_{1}$-Generation in der Farbe identisch mit I, bzw. Fasciata bleibt. Bei intermediären Befunden kann die Ursache das Fehlen einer genauen Untersuchung oder relativ heterozygotes Elternmaterial mit breiter Färbungsveranlagung sein. Im Falle KuvzE (1956) handelt es sich deutlich um sehr heterozygotes Ausgangsmaterial. Bei WATsON (I927) war es vermutlich eine nicht mit Erfolg durchgeführte künstliche Besamung, wobei die Königin sich nachträglich mit schwarzen Drohnen gepaart hat.

In Bezug auf die Vererbung der Farbmusterung bei der $F_{\mathbf{1}}$-Generation der Arbeitsbienen ist aus diesem Versuch zu ersehen, dass andere genetische Regeln als bei den Drohnen von $9 q$ der $\mathrm{F}_{1}$-Generation reziproker Kreuzungen herrschen. ROBERTS (I950) und KULINĊEVIC் (I966) fanden Übergangsmuster bei BastardDrohnen von der einen zur anderen Farbrasse, was die frühere Auffassung über die I : I-Spaltung widerlegt. Fine Anhäufung von Zeichnungsmustern im Bereich der beiden Ellternrassen mit deutlichen Zeichen der Bastardierung der Drohnen ist von KULINČEVIĆ (I966) nachgewiesen. Bei Arbeitsbienenmustern der reziproken Kreuzungen $C a \times L$ haben wir ein starkes Dominieren der $L$ gelben Pigmentierung und keine breite Streutung.

Beide Fälle sprechen nicht für einen einfachen monofaktoriellen Lirbgang. Die schon erwöhnte, unbestimmte Pigmentierungszone am Seitenlappen der Tergite bei Reziproken soll nicht übersehen werden, aber wie gesagt, beeinflusst sie nicht die Farbmusterung einer Bastard-Arbeitsbiene.

Die erheblich grössere Streuung bei der Gesamt-Tergitfläche von $L$ im Verhältnis zur niedrigeren Streuung bei der Gesamtdunkelfäche kommt wahrscheinlich daher, dass die Grösse der Tergite durch Umweltbedingungen beeinflusst werden kann. Is ist anzunehmen, dass sich die Vererbung der Tergitmusterung unabhängig von der Tergitgrösse entwickelt. I)ie Gesamtfläche vererbt sich ansonsten in diesem Versuch intermediär, was mit Befunden an anderen Merkmalen iibereinstimmt.

Diese Eirgebnisse sind von besonderer Bedeutung für genetische Untersuchungen bei Bienen, weil wir bei Kreuzungen zwischen $L$ und anderen dunklen Rassen, ebenfalls zwischen Fasciata und dunklen Rassen Bastarde erwarten können, die ohne detaillierte Untersuchungen nach Farbmusterung nicht zu unterscheiden sind. Deswegen muss man bei der Beurteilung der Farbmuster vorsichtig sein, um jede mögliche Verbastardierung auszuschliessen, weil die Gewinnung und Frhaltung von reinen Linien für Bienenzucht sehr wichtig sind. Natürlich sind diese Unterscheidungen nicht möglich, wenn nur heterozygotes Material zur Verfïgung steht oder eine Paarung mit nicht uniform-gefärbten Drohnen stattgefunden hat. 


\title{
RÉSUMÉ
}

\author{
HÉRÉDITF́, DE LA PIGMENTATION CHEZ LES OUVRIERES D' APIS MELLIFICA " I. \\ ET ISSLES DE CROISEMENTS ENTRE RACES
}

L'auteur a mesuré et interprété les dessins colorés des $3^{\mathrm{e}}, 4^{\mathrm{e}}$ et $5^{\mathrm{e}}$ tergites chez des ouvrières des races Carnica et Ligustica ou issues de croisements réciproques.

Les dessins des souches initiales ont été rangés dlans les classes extrêmes du système de GoETze : Carnica dans la classe I et Ligustica dans la classe ().

Les dessins de la génération $\mathrm{F}_{1}$ correspondent en totalité it la classe 8. Quoique les métisses se trouvent dans la classe voisine de Ligustira, il y a entre les deux une différence notable qui s'appuie sur des comparaisons statistiques. La part de pigmentation sombre che $z$ les animaux issus de croisements réciproques augmente en moyenne de $10,7 \mathrm{p}$. 100, le $3^{\mathrm{e}}$ tergite de $8,0 \mathrm{p}$. 100, le $4^{\mathrm{e}}$ tergite de I 3,8 p. roo et le $5^{\text {e }}$ de 10,7 p. 100.

Ies croisements réciproques ne se distinguent pas entre eux par le dessin. Il n'y a pas trace de matroclinie dans l'hérédité des dessins colorés. Chez les animaux issus de croisements réciproques les dessins sont très uniformes et présentent un écart type qui est un peu plus important que che\% les races Carnica ou Ligustica. I ne dominance de la pigmentation jaune est très nette.

Che\% les métisses on a remarqué sur les lobes latéraux une zone intermédiaire mal délimitée; elle n'a pas d'influence sur le dessin coloré bien qu'elle soit une conséquence du croisement.

L'hérédité des bandes sombres semble tout à fait indépendante de la surface totale des tergites. Ceci est très net chez la race Iigustica. (hez cette dernière la surface totale des tergites présentajt un écart type beaucoup plus important que la surface sombre du dessin coloré, ce qui est dû vraisemblablement aux conditions ambiantes.

\section{SUMMARY}

THE INUERITANCE OF PIGMENTATION IN WORKLRS OF "APIS MELLIFICA DESCENDANTS OF CROSSINGS BETWEEN BREEDS

The author meisured and interpreted the colour patterns of the third, fourth, and fifth tergites of workers of the breeds Carnica and Ligustica and of the descendants of reciprocal crossings.

The patterns of the initial strains fell into the extreme classes of the (ioetze system : Carnica in class I and Ligustica in class 9.

The patterns of the $F_{1}$ generation corresponded perfectly with class 8 . Although the hybrids fell into the class adjacent to that of the breed Ligustica, there was a distinct difference between the two groups when compared statistically.

The dark pigmented part of the hybrids was higher on average by $10.7 \mathrm{p} .100$, the third tergite by 8.0 p. Ioo, the fourth tergite by 13,8 p. 100, and the fifth by 10,7 p. I00.

The offspring of two reciprocal crossings could not be distinguished from each other ; there was no matroclinous link in the inheritance. The patterns were very uniform and showed a standard deviation which was a little greater than in the parent breeds. There was a very clear dominance of yellow pigmentation.

A badly delimited intermediate zone was noted on the lateral lobes of the hybrids; this had no effect on the colour pattern although it may be a consequence of the crossing.

The inheritance of the dark bands seems quite independant of the total surface of the tergites. This is very clear in the breed Ligustica. In this breed the total surface of the tergites shows a standard deviation which is much more significant than the dark surface of the colour pattern, this probably being due to the environment. 


\section{ZUSAMMENFASSUNG}

Es wurden die Farbmuster der 3., 4. und 5. Tergit bei $A$ rbeitsbienen $\operatorname{der} C a$ und $L$ Rasse (Linien) und ihre reziproken Kreuzungen gemessen und ausgewertet.

Die Farbmuster des Ausgangsmaterials wurden in GOETZES 9-Klassen-System an den äussersten Grenzen eingestuft, $\mathrm{Ca}$ in Klasse $\mathrm{I}$ und $L$ in Klasse 9 .

Die Farbmuster der $F_{1}$-Generation sind vollständig in Klasse $8 \mathrm{zu}$ finden. Obwohl die Bastarde in der benachbarten Klasse von $L$ eingeordnet sind, zeigt sich ein deutlicher Unterschied von $L$, welcher durch statistische Vergleiche festgestelit wurde. Der Anteil von dunkler Pigmentierung bei Reziproken vergrössert sich durchschnittlich um I0,7 p. I00, bei dem 3. Tergit um 8,0 p. I00, dem 4. Tergit um I3,8 p. Ioo und dem 5. Tergit um 10,7 p. 100.

Die reziproken Kreuzungen unterscheiden sich untereinander nicht in der Musterung. Eine Matroklinie trat bei der Vererbung des Farbmusters nicht in Erscheinung. Die Farbmuster bei Reziproken sind sehr einheitlich und zeigen die Streuung, die etwas grösser als bei der $\mathrm{Ca}$ oder $I$. Rasse ist. Ein Dominieren der gelben Pigmentierung ist klar erkennbar.

Bei Bastarden wurde eine unbestimmte Ửbergangszone am Seitenlappen festgestellt ; sie beeinflusst nicht das Farbmuster, obwohl sie ein Produkt der Kreuzungen ist.

Die Vererbung von dunklen Streifenffächen scheint ganz unabhängig von den Gesamt-Tergitflächen zu sein. Das zeigt sich sehr deutlich bei der $L$ Rasse. Die Gesamtfläche der Tergite bei dieser Rasse hatte eine viel grössere Streuung als die dunkle Farbmusterfäche, was wahrscheinlich durch Umweltbedingungen entstanden ist.

\section{LI'TERATUR}

Armbruster L., 1923. Wie untersucht man l3ienenstämme und Bienenkreuzungen auf ihre liarbe? Arch. Bienenkunde, $\mathbf{5}(4), 97$-I 30 .

Arusbruster L., Nachtscieis Il., Roemer 'T., i9i6. Die Hymenopteren als Studienobjekt azygoter Vererbungserscheinungen. Induklive Abstammungs-und Vererbungslehre, 17, 273-355.

Drescier W., Kulincević J., 1967. (Manuskript unveröffentlicht) Die Vererbung der Rüssellänge bei reziproken Kreuzungen Apis mellifica L.

Eir. BAnby M. A., 1965. Die Vererbung der Unterleibwechselbeziehung bei den Mestizen der CarniolischAgyptischen. Apimondia 20. internation. Kongr., Bukaresl.

Farcas A., 1938. Contributioni la Studial Culorii albineloa. Bul. Fakullati de agronomie Bukaresti, 7, 4-9.

GoeTzE G., I930. Variabilitäts- und Züchtungsstudien an der Ionigbiene mit besonderer Berücksichtigung der Langrüsseligkeit. Arch. Bienenkunde, 11 (5-6), 185-279.

GOETZE G., i936. Technik und Lirgebnisse, Lirbversuche mit der IIonigbiene. Deutscher Imkerführer (10), $68-7.4$.

GOETZE G., 1964. Die Honigbiene in natürlicher und küntslicher Zuchtauslese. Teil I und II, Paul Parey, IIamburg.

KrCzAL II., I952. Untersuchungen zur Variabilität von Flügelindex und Rüssellänge bei der Honigbiene Z. Bienenforsch. 1 ( (1 1 ), 225-234.

KuniNCÉviC' J., 1960. Die Phänoanalyse der Abdominal-Tergite von Drohnen verschiedener greographischer Rassen der Honigbiene (Apis mellifica L). und ihrer Kreuzungen. Ann. Abeille, 9 (2), I I $5^{-1} 5_{2}$.

KUNZE G., I956. Untersuchungen über die Variabilität zweier Rassen von Apis mellifica $\mathrm{L}$. sowie ihrer kreuzungen unter Anwendung der graphischen Form und Korrelations-analyse. Morphol. Ökol. Tiere, 44, $269-328$.

LaIdLaW H. H., EL Banby M. A., ig62. Inhibition of yellow body colour in the honey bee. Heredity 3 (4), I 7 I-I 73 .

Mackensen $0 ., 195^{6}$. Some effects of inbreeding in the honey bee. 16e inlernation. Beekeep. Kongr. Wien.

Mrchailof A. S., I930. On the inheritance of the colour and some quantitative characters in $F_{1}$ in the honey bee. Opilnaja Paseka, 21 5-228.

NeWELl W., I915. inheritance in the honey bee. Science, 41 (1049), 218-219).

Not.A. W. J., I937. Bee Breeding, U. S. Depl. Agric. Yearbook pP. 1936-1418. 
Roberts W. C., r950. (Dissertation) Summary and Conclusions (Univ. of Wisconsin for Ph. D. Examina tion).

Roberts W. C., Mackensen O., I95I. Breeding improved honey bees. Amer. Bee J., 91, 292-294, 328-330; $3^{82-384}, 4 \mathrm{I} 8-42 \mathrm{I}, 473-475$.

Rutrner F., r949. Zur Genetik der Honigbiene (Apis mellifica) ihre Probleme und Möglichkeiten. Portugalae Acta Biologica, Ser. A, 232-257.

Ruttner F., I953. Über die Vererbung einiger Rassenmerkmale bei der Honigbiene (Apis mellifica). Osterr. Zool. Z., 4, I-2, I83-I90.

RutTner F., I957. Die Sexualfunktionen der Homgbienen im Dienste ihrer sozialen Gemeinschaft. $Z$. vergleich. Physiol., 39, 577-600.

Ruttner F., Mackensen O., 1952. The Genetics of the honey bee. Bee World, 33 (4), 53-62 and 5, 7 I-79. Sladen F. W., I9I3. Queen-rearing in England. ze éd. 86 p., London.

Vecchi A., 1927. Sulla Distributione Geographica dell' Apis mellifica Ligustica Spin. in Italia. Boll. del. Labor. die Zoolog. Generell. a agraria, zo.

WATSON L. R., 1927. Controlled mating in the honey bee. Iowa Siate Apiarist, 36-41.

ZANDER E., I923. Beiträge zur Variabilität und Vererbung bei der Honigbiene. Erlanger Jahresbuch Bienenkde, 1, 5-66. 